\title{
Body size preferences in the pot-bellied seahorse Hippocampus abdominalis: choosy males and indiscriminate females
}

\author{
Beat Mattle • Anthony B. Wilson
}

Received: 17 March 2008 /Revised: 12 May 2009 / Accepted: 31 May 2009 /Published online: 26 June 2009

(C) Springer-Verlag 2009

\begin{abstract}
Male seahorses (genus Hippocampus) provide all post-fertilization parental care, yet despite high levels of paternal investment, these species have long been thought to have conventional sex roles, with female mate choice and male-male competition. Recent studies of the potbellied seahorse (Hippocampus abdominalis) have shown that sex-role reversal occurs in high-density female-biased populations, indicating that male mating preferences may lead to sexual selection on females in this species. Egg size, egg number, and offspring size all correlate positively with female body size in Hippocampus, and by choosing large mating partners, male seahorses may increase their reproductive success. While male brood size is also positively correlated with body size, small $H$. abdominalis males can carry exceptionally large broods, suggesting that the fecundity benefits of female preference for large partners may be limited. We investigated the importance of body size in reproductive decisions of $H$. abdominalis, presenting focal individuals of both sexes with potential mating partners of different sizes. Mating preferences were quantified in terms of time spent courting each potential partner. Male seahorses were highly active throughout the mate-choice trials and showed a clear behavioral preference for large partners, while females showed significantly lower levels of activity and equivocal mating preferences. The strong male preferences for large females demonstrated here suggest that sexual selection may act strongly on female body size in wild populations of $H$. abdominalis,
\end{abstract}

Communicated by C. St. Mary

B. Mattle $\cdot$ A. B. Wilson $(\bowtie)$

Zoological Museum, University of Zurich,

Winterthurerstrasse 190,

8057 Zurich, Switzerland

e-mail: tony.wilson@zm.uzh.ch consistent with predictions on the importance of female body size for reproductive output in this species.

Keywords Body size - Mate choice - Sexual selection · Sex roles $\cdot$ Syngnathidae

\section{Introduction}

Body size is an important trait in mate-choice decisions of most animal species, and a preference for larger partners is often found (e.g., Herdman et al. 2004; Berglund et al. 2006; Byrne and Rice 2006). This preference is thought to reflect the direct benefits of large body size in territory defense, resource acquisition, and reproduction in addition to genetic benefits associated with mating with a largebodied partner. Although most animals have conventional sex roles, where males compete for mating opportunities and females are choosy, sex-role reversed mating systems also exist, in which females compete among each other for access to males and males are choosy (Andersson 1994). In both systems, the relative parental investment of the sexes in their young is seen as a key variable underlying sexual selection, and as male investment in their young increases, female competition for males is expected to intensify (Trivers 1972). While parental investment strongly influences mating competition, optimal mating behavior and sex roles also depend on ecological factors such as the spatial and temporal distribution of receptive mates, factors which influence the operational sex ratio (Emlen and Oring 1977; Shuster and Wade 2003). It is important to recognize that while mate-choice decisions are commonly considered to be one-sided and inflexible, mate choice may often be mutual both in species which are considered to have conventional sex roles and in those with sex-role reversal 
(Johnstone et al. 1996; Bergstrom and Real 2000; Kokko and Johnstone 2002). Recent empirical evidence indicates that sex roles may shift rapidly within a single breeding season according to ecological resources and availability of partners (Forsgren et al. 2004; Kolm 2004).

Seahorses have a unique mode of reproduction: male pregnancy. Male seahorses have a fully enclosed brood pouch into which females transfer their eggs prior to fertilization. After fertilization, embryos implant in the brood pouch wall, where they are osmoregulated, aerated, and potentially nourished until their release as fully developed independent juveniles (reviewed in Stölting and Wilson 2007). Notwithstanding this high level of paternal care, Hippocampus seahorses have long been thought to have conventional sex roles (Wilson et al. 2003; reviewed in Foster and Vincent 2004), and observational data from aquaculture populations of the pot-bellied seahorse (Hippocampus abdominalis) indicate that male-male competition and female choice are also found in this species (Woods 2000). In contrast to these reports, a recent field study described sex-role reversal in female-biased populations of $H$. abdominalis (Wilson and Martin-Smith 2007), with high levels of female intrasexual competition and male mate choice. These results indicate that sex roles in $H$. abdominalis may be flexible and that mutual mate choice may operate in this species. Both Martin-Smith and Vincent (2005) and Wilson and Martin-Smith (2007) found that female $H$. abdominalis were larger than males in all populations surveyed, and female-biased size dimorphism has also been found in the Western Australian seahorse (Hippocampus subelongatus; Kvarnemo et al. 2007). Recent work on this species identified a significantly positive sexual selection differential on female body size, with increased mating success of large-bodied females, but no evidence for differential reproduction associated with body size differences in males (Kvarnemo et al. 2007), suggesting that female-biased sexual size dimorphism in seahorses may be driven in part by sexual selection.

Clutch, egg, and offspring size typically increase with female body size in ectotherms (reviewed in Blanckenhorn 2005), and female body size is also positively correlated with both offspring number (Woods 2007) and size in $H$. abdominalis (Vincent 1990), indicating the clear fecundity benefits of large female body size in seahorses. While large females have clear advantages over smaller individuals in terms of fecundity, the relationship between male body size and fecundity in $H$. abdominalis is somewhat more complicated. Woods (2005) found a positive correlation between the number of juveniles per brood and body size of the brooding male in a wild population of pot-bellied seahorses, supporting a link between male body size and fecundity. However, despite this general correlation between male body size and brood size, Woods $(2000,2005)$ found that large broods could also be carried by small males. If size-assortative mating occurs in H. abdominalis, a pattern found in other seahorse species (Vincent and Giles 2003), the fact that larger broods are carried by large males may simply reflect the higher fecundity of their mating partners (Woods 2000).

While male body size may not directly influence maximum fecundity in $H$. abdominalis, juveniles of large males might benefit from having a higher growth rate and/or higher survival due to better resource allocation during early development. Although mean juvenile length and brood size are negatively correlated in most seahorses (Vincent 1990), no such correlation is evident in $H$. abdominalis (Woods 2005). H. abdominalis have larger brood pouches than other seahorses (Vincent 1990), and male H. abdominalis may be large enough to provide an optimal breeding environment for all embryos, even when clutch size is large. Thus, while female body size has a significant influence on fecundity in H. abdominalis, the fecundity benefits of large male body size remain equivocal.

Given the documented flexibility of sex roles in $H$. abdominalis (Woods 2000; Wilson and Martin-Smith 2007), we explored the role of body size in both female and male mate-choice decisions, using a two-choice experimental design. We tested the hypothesis that female pot-bellied seahorses show no body size preference due to high fecundity of both large and small males (Woods 2000; reviewed in Foster and Vincent 2004) and that males prefer large females, as female body size is known to be the key determinant of both offspring number and size in $H$. abdominalis (Vincent 1990).

\section{Materials and methods}

H. abdominalis populations are distributed throughout the temperate marine waters of New Zealand and south-eastern Australia and are currently listed as threatened under the Convention for International Trade in Endangered Species (Lourie et al. 1999). Sexually mature, 6-month-old, $H$. abdominalis individuals were obtained from a captive breeding facility in Tasmania (Seahorse Australia, Beauty Point) in August 2006. Founder seahorses of this population were collected from a variety of locations around Tasmania and have been bred in aquaculture for seven generations (Wilson and Martin-Smith 2007). Animals in the captive breeding facility are mated in large communal $(2,100 \mathrm{~L})$ breeding tanks with approximately 50 males and females per tank, allowing free mate choice among the reproductive population (R. Hawkins, pers. comm.). Microsatellite analyses indicate that genetic diversity of aquaculture animals is comparable to that of wild populations (Mattle and Wilson, unpublished data). After arrival, males and females were kept separately to avoid potential pair 
bonding before mate-choice trials (see "Introduction"). Stock tanks were connected to a central seawater circulation system and contained identical artificial plastic plants as holdfasts and shelters. Seahorses were fed ad libitum three times each day with frozen Artemia salina and Mysis relicta. Animal husbandry and experimental procedures were approved by the Veterinäramt of Kanton Zurich (Permit 185/2006).

Seahorses were anesthetized by placement for $5 \mathrm{~min}$ in $10 \mathrm{~L}$ of a $40.5 \mathrm{mg} / \mathrm{L}$ solution of AQUI-S (isoeugenol; AQUI-S, Lower Hutt, New Zealand) and tagged with a specific four-color visible implant fluorescent elastomer tag (Northwest Marine Technology, Shaw Island, USA), according to Woods and Martin-Smith (2004). Consistent with the results of Woods and Martin-Smith (2004), no adverse effects or mortality occurred as a result of anesthesia and tagging. During anesthesia, a digital photo of each animal was taken in standardized lateral orientation, and the wet weight of the seahorse was measured. The standard length (SL; Lourie 2003) of each seahorse was calculated with tpsDig v2.05 (Rohlf 2004) using digital landmarks along the lateral body axis. Seahorses were divided into four size classes: small females (mean $\pm \mathrm{SD}$ ) $19.25 \pm 0.62 \mathrm{~cm} \quad(n=15)$, large females $21.89 \pm 1.01 \mathrm{~cm}$ $(n=15)$, small males $18.27 \pm 1.21 \mathrm{~cm}(n=15)$, and large males $21.52 \pm 0.8 \mathrm{~cm}(n=15)$. Standard length measurements were normally distributed (Shapiro-Wilk test: $P>$ 0.067 for each size class), and the assumption of equal variances of the four size classes could not be rejected (Bartlett test: $\chi_{(3)}{ }^{2}=5.80, P=0.122$ ). Female seahorses $(20.57 \pm 1.57 \mathrm{~cm}, n=30)$ were significantly larger than males $(19.89 \pm 1.94 \mathrm{~cm}, n=30)$, and large individuals were significantly larger than small individuals in both sexes (two-way ANOVA: sex: $F_{1,56}=7.19, P=0.01$; size: $F_{1,56}=$ 143.25, $P<0.001)$, with no interaction between these factors $\left(F_{1,55}=1.50, P=0.23\right)$. The SL of all experimental seahorses $(n=59)$ was positively correlated with wet weight $\left(F_{58}=37.66, r^{2}=0.398, P<0.001\right)$. However, as SL was only a marginal predictor of weight, the influence of both SL and body weight in mate-choice decisions was investigated.

Water parameters were kept constant throughout the experimental period (16 January-16 February 2007; temperature $20.0 \pm 0.3^{\circ} \mathrm{C}, \mathrm{pH} 8.1 \pm 0.0$, salinity $31.5 \pm 0.8 \mathrm{ppt}$ ), and the light regime was maintained at 14:10 hours (light to dark). Mate-choice experiments were conducted in two choice tanks $(55 \times 55 \times 75 \mathrm{~cm}, \approx 210 \mathrm{~L}$; Fig. 1$)$. Choice tanks were located in the same room as the stock tanks and were connected to the same seawater circulation system, ensuring constant water conditions in stock and experimental tanks. Choice tanks were divided into three sections (Fig. 1), with an opaque divider separating the two stimulus animals and a clear plexiglass divider separating the

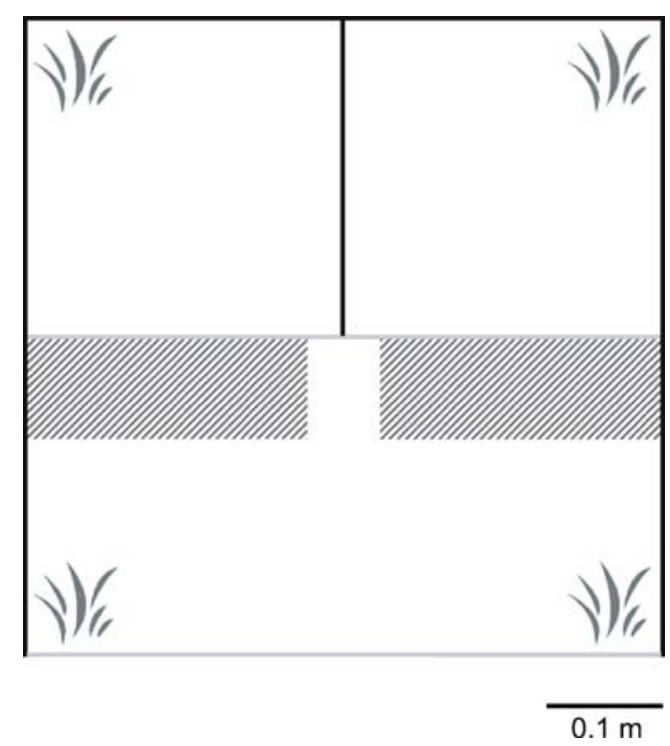

Fig. 1 Schematic experimental tank design (top view) showing location of plastic plants in each corner, dividing walls, and the defined preference zone (hatched areas). Black-colored walls were opaque and gray-colored walls were clear

stimulus animals from the focal individual. A series of small holes $(5 \mathrm{~mm}$ diameter) were made $1 \mathrm{~cm}$ from the bottom of the clear divider, maintaining a constant flow of water from the two stimulus fish compartments to the focal fish. No visual or chemical exchange took place between the stimulus animals. Test tanks were illuminated by overhead fluorescent tubes, providing similar illumination levels to stock tanks (50.3 vs. $57.1 \mathrm{~lx}$ ).

Seahorses were transferred to choice tanks between 1600 and 1700 hours on the day before the experiment to allow acclimatization and were not fed during the settling period or trial. Mate-choice experiments were conducted from 0800 to 0930 hours (total time, 5,400 s) initiated by the onset of the artificial light phase. Seahorses are reproductively most active during the first hours of daylight (Vincent and Sadler 1995; Masonjones and Lewis 1996).

Each seahorse was offered a choice between two seahorses of the opposite sex belonging to different size classes. The compartment with the focal fish was located in the front half of the tank, facing two quarter compartments (Fig. 1). In order to harmonize the difference in size between the two stimulus fish, individuals were paired according to their size class rank: The SL difference between the two stimulus fish was $3.23 \pm 0.59 \mathrm{~cm}(n=29)$ for male-stimulus fish and $2.62 \pm 0.53 \mathrm{~cm}(n=30)$ for female-stimulus fish. The side of the compartment for the two stimulus fish, triplet groupings, and experimental tank were all randomized, after excluding previously used animals. Thus, every individual seahorse was involved in three trials, twice as one of two stimulus animals and once as the focal animal, with a minimum time between trials of 
two days $(x=2-11$ days). One small female seahorse died 4 days after its trial as focal fish and was substituted in the two following trials with two different females of the same size class. This individual was excluded from all statistical analyses. A second small female was used three times as a stimulus animal because of confusion with the individual color code. However, no individual of either sex had visual contact to the same individual at any point during the trials. After each trial, seahorses were transferred to stock tanks corresponding to their class, maintaining separation between experienced and inexperienced fish.

Observations were recorded with two digital video cameras per test tank (ABUS, Wetter, Germany), which were connected to a computer generating films in real time. One camera was mounted above the tank and the other in front of the focal compartment. Top view records were analyzed by eye, scoring time spent showing reproductive behavior in either preference zone $(7.5 \times 22.5 \mathrm{~cm}$; Fig. 1). Preference zones were distinguished by a checkered grid ( $2.5 \mathrm{~cm}$ side length per square) covering the bottom of the experimental tanks.

Frontal view records were scanned for courtship behaviors, which have been well characterized in H. abdominalis and occur in an established sequence prior to reproduction (Woods 2000, Table 1). Behavioral preference was scored if the focal fish entered a preference zone with its full head length and showed behavior consistent with reproductive interest (Table 1). The values for behavioral preference $(B)$ of the focal animal were quantified in terms of the relative proportion of time it showed courtship behavior within the preference zone in front of the large individual compared to the total time showing courtship behavior in either preference zone. Consequently, a value for $B$ above 0.5 indicates that more time was spent courting the large

Table 1 Ethogram of courtship behavior for the pot-bellied seahorse, Hippocampus abdominalis

\begin{tabular}{ll}
\hline $\begin{array}{l}\text { Sequence } \\
\text { order }\end{array}$ & Description \\
\hline 1 & $\begin{array}{l}\text { Pumping: male inflates the brood pouch with water } \\
2\end{array}$ \\
3 & $\begin{array}{l}\text { Interaction: pair oriented against each other } \\
\text { the head tucked down and dorsal and pectoral } \\
\text { fins rapidly fluttering }\end{array}$ \\
4 & $\begin{array}{l}\text { Promenading: pair in tandem; occurs in short bursts } \\
5\end{array}$ \\
6 & $\begin{array}{l}\text { Pointing: pointing the snout upward } \\
\text { the water surface }\end{array}$
\end{tabular}

Modified from Woods (2000). These behaviors occur in a wellcharacterized sequence prior to reproduction (Masonjones and Lewis 1996; Woods 2000). The behaviors pumping and jack-knifing are only performed by male seahorses partner, while values below 0.5 indicate that more courtship behavior was shown in front of the small partner. As behavioral preference was scored as a proportion $(0-1)$, all preference values were arcsine-transformed prior to analysis. Following transformation, preference values were normally distributed (Shapiro-Wilk test: $P>0.214$ for female and male preferences).

To consider the possibility that body size of the focal animal and/or assortative mating preferences influenced observed behaviors, two GLMs were constructed, using both SL and body weight as separate measures of body size. These models incorporated body size of the focal individual and assortative preference scores as continuous predictors and sex as a fixed factor. Assortative preference scores were calculated as the absolute value of the body size difference between the focal and large stimulus individual divided by the difference in body size between the focal and small stimulus animal. Assortative preference scores were log-transformed prior to analysis.

The fit of these two models to the observed data was calculated using second-order Akaike information criterion scores $\left(\mathrm{AIC}_{\mathrm{c}}\right.$, Burnham and Anderson 1998). The relative performance of the two models is reported here as Akaike weights $\left(\mathrm{AIC}_{\mathrm{w}}\right)$. All statistical analyses were performed using SPSS v13.0 (Chicago, IL, USA).

\section{Results}

Seahorse courtship behavior follows a clear sequence prior to mating and involves a series of increasingly intense interactions between mating partners (Table 1). The following behaviors were observed during mate-choice experiments: "pumping" (25 males), "interaction" (15 females, 20 males), "jack-knifing" (12 males), "promenading" (three females, five males), and "pointing" (one female, three males; Table 1). Promenading was only possible for a short burst of swimming due to the restricted area for interaction between the fish in the compartments. "Rising" was theoretically possible given the experimental tank design, but was not observed in any of the trials. While pointing has previously only been reported in males (Woods 2000), a female H. abdominalis initiated pointing in one of the experimental trials performed here. A conspicuous behavior performed by some pumping male seahorses was observed while they were emptying their brood pouch: While passively sinking down from the top of the water column to the bottom, these males dilated their brood pouch opening by straightening their tail and folding their brood pouch.

Fourteen focal females and seven focal males did not enter the preference zones at any time during their trial (i.e., no interest shown). Significantly fewer females than males 
entered the preference zone $\left(t_{57}=-2.04, P=0.046\right)$. Males spent significantly more time in the preference zones than did females $\left(t_{57}=-3.63, P<0.001\right.$; Fig. 2$)$, even after excluding individuals who never entered the preference zone at any point during the trial $\left(t_{36}=-2.98, P=0.005\right)$. The five smallest females did not enter the preference zone at any time during their experimental trials, suggesting that female interest in reproduction may be correlated with body size. Consistent with this hypothesis, the total time a focal female spent within the preference zone correlated positively with her SL $\left(F_{1,27}=5.07, r^{2}=0.16, P=0.033\right)$. The same comparison was not significant for males $\left(F_{1,28}=0.70\right.$, $\left.r^{2}=0.02, P=0.411\right)$, indicating no correlation between male body size and activity levels during the trials. One or both stimulus animals were at the divider in the majority of trials ( 28 of 29 focal-female trials vs. 26 of 30 focal-male trials), and we were interested to see whether the size of the focal animal influenced reproductive interest of the stimulus animals. Stimulus animals were more active in focal-female trials $\left(F_{1,56}=4.13, P=0.046\right)$, but the size of the focal individual did not influence stimulus animal interest $\left(F_{1,56}=2.87, P=0.096\right)$. Thus, as small-bodied focal animals had ample opportunity to demonstrate reproductive interest, the lack of reproductive behavior in these individuals cannot be attributed to the behavior of their potential mating partners.

Animals which did not (1) visit both sides of the experimental tank, (2) enter the preference zone, and (3) show courtship behavior as either focal or stimulus animals were not included in further statistical analyses, yielding a final number of ten trials involving focal females and 16 trials involving focal males.

The behavior interaction was shown by these females for $421.50 \pm 487.83 \mathrm{~s}(n=10)$, promenading for $18.0 \pm 5.66 \mathrm{~s}$

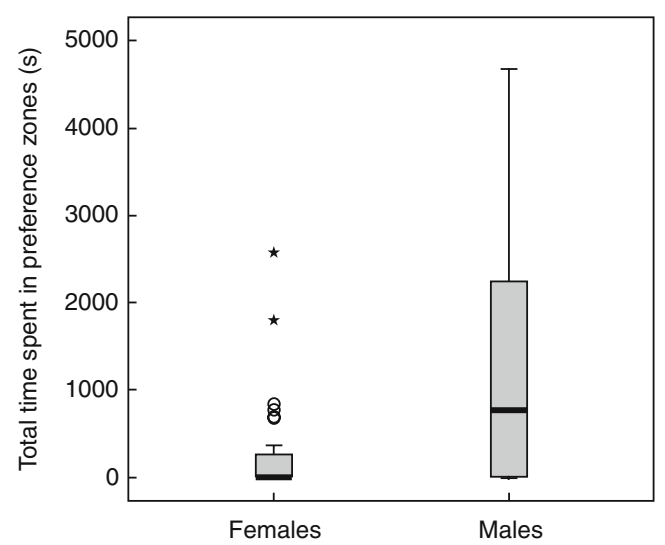

Fig. 2 The total time female $(n=29)$ and male $(n=30) H$. abdominalis spent in the preference zones. Males spent significantly more time in the preference zone $(P=0.001)$. Open circle values more than 1.5 box lengths over the 75 th percentile; star values more than three box lengths over the 75 th percentile. The total experimental trial period was $5,400 \mathrm{~s}$ $(n=2)$, and pointing for $3.0 \mathrm{~s}(n=1)$. Females who showed more than a single behavior $(n=2)$ displayed these behaviors according to their expected sequence (Table 1). Within the focal-male trials, the behavior pumping was observed for $1,927.33 \pm 1,319.01 \mathrm{~s}(n=15)$, interaction for $560.33 \pm 456.31 \mathrm{~s}(n=15)$, jack-knifing for $74.67 \pm 74.14 \mathrm{~s}$ $(n=9)$, promenading for $17.00 \pm 12.65 \mathrm{~s}(n=4)$, and pointing for $6.67 \pm 5.51 \mathrm{~s}(n=3)$. The established sequence of behaviors was shown by 14 focal males. One male showed only pumping, while a single male showed interaction with both stimulus fish despite no obvious pouch pumping.

The total time showing courtship behavior in the preference zones varied considerably among individuals. Females spent an average of $421.50 \pm 487.83$ s showing courtship behavior in the preference zones, while males spent significantly more time showing courtship behavior $\left(1,811.63 \pm 1,355.74 \mathrm{~s}, F_{1,24}=9.61, P=0.005\right)$.

To address the question of whether experimental seahorses preferred larger partners, the null hypothesis of no preference (i.e., $B=0.5$ ), was tested with one-sample $t$ tests. While females showed no preference for large males $\left(B=0.64 \pm 0.40,95 \%\right.$ CI $\left.0.355-0.929, t_{9}=0.98, P=0.351\right)$, males showed a significant preference for large partners $\left(B=0.71 \pm 0.27,95 \%\right.$ CI $0.563-0.849, t_{15}=3.06, P=0.008$; Fig. 3).

Neither the body size of the focal animal nor assortative mating preferences for either SL or body length explained a significant proportion of the observed preference behaviors. Focal animal SL and assortative preference scores were both non-significant predictors of preference (focal animal SL $F_{1,22}=2.60, P=0.121$; assortative preference score $\left.F_{1,22}=2.23, P=0.149\right)$ with no interaction between these factors (focal animal $\mathrm{SL} \times$ assortative preference score $F_{1,21}=0.83, P=0.111$ ). Similarly, weight of the focal animal and assortative preference based on weight also did not explain a significant proportion of the observed behaviors (focal animal weight $F_{1,22}=0.07, P=0.798$; weight-based assortative preference $\left.F_{1,22}=0.02, P=0.901\right)$ with no interaction between these factors (focal animal weight $\times$ weight-based assortative preference $F_{1,21}=0.00, P=0.955$ ).

While neither GLM model explained a significant proportion of the observed behavioral preferences, the GLM using SL as a measure of body size fit the observed data better than did that constructed using body weight $\left(\mathrm{AIC}_{\mathrm{c}}\right.$ : SL model $=36.98$, weight model $=40.35 ; \mathrm{AIC}_{\mathrm{w}}$ : SL model $=0.844$, weight model $=0.156$ ), yielding an evidence ratio (length/weight) of 5.41 .

\section{Discussion}

The results presented here indicate that mating behaviors in the pot-bellied seahorse ( $H$. abdominalis) differ significant- 


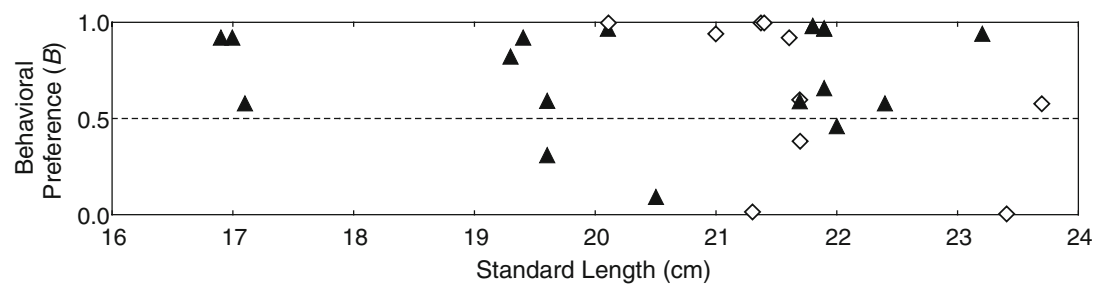

Fig. 3 The individual behavioral preferences $(B)$ of female (diamonds, $n=10$ ) and male (triangles, $n=16) H$. abdominalis that showed reproductive behavior in the preference zones during mate-choice experiments. Behavioral preference $(B)$ is defined as the relative proportion of time a focal individual spent courting the large partner

ly between males and females. While males were highly active and showed a clear behavioral preference for large partners during the mate-choice trials, female activity was lower and mating preferences were equivocal. These results are consistent with predicted fecundity benefits of female body size. A similar pattern was reported in a mate-choice experiment with the sex-role-reversed pipefish Syngnathus typhle (Berglund et al. 2006). While large females preferred large partners in S. typhle, the choice of small females was randomly distributed, and males had a stronger preference for large partners than did females. Both studies indicate that sexual selection in natural populations of these species may act more strongly on female than male body size.

Consistent with this pattern, a recent field study of the Western Australian seahorse, H. subelongatus, found that female body size is under stronger sexual selection than is male size. Mated females in this study were significantly larger than unmated individuals, while no size difference was found between mated and unmated males (Kvarnemo et al. 2007). If these differences in sexual selection differentials are consistent across species, sexual selection pressures may explain the sexual size dimorphism found in populations of $H$. abdominalis, where females are larger than males (Wilson and Martin-Smith 2007).

Kvarnemo et al. (2007) were unable to determine whether size differences in mated and unmated females were due to intrasexual competition among females or male preference for large female body size. The results presented here, showing male preference for large females, coupled with field observations of aggressive female-female interactions during mating (Wilson and Martin-Smith 2007) suggest that sexual dimorphism may arise via a combination of these two factors. Direct measurements of mating competition and sexual selection differentials in the wild, coupled with behavioral observations of mating competition under experimental conditions, may offer a means to determine the relative importance of intrasexual competition and mate choice in reproductive interactions.

Recent research has found evidence of size-assortative mating in wild populations of two seahorse species (Jones compared to the total time it spent courting both partners. Consequently, preference values above 0.5 indicate that more reproductive activity was shown toward the large partner, while values below 0.5 indicate that more activity was shown toward the small partner

et al. 2003; Vincent and Giles 2003). In contrast to these studies, we found no evidence of size-assortative mating preferences in either male or female $H$. abdominalis on the basis of SL or body weight. While reproductive seahorses are typically found as breeding pairs, $H$. abdominalis form large breeding aggregations in the wild (Martin-Smith and Vincent 2005). If reproductive individuals aggregate prior to pairing in other species, inferred assortative mating in wild populations could occur as a consequence of age structuring in these aggregations and not necessarily reflect active positive assortment.

Male preference for large partners was predicted due to the higher fecundity of larger females. As gravid females often have swollen abdominal areas after egg hydration (Woods 2000), the roundness of a female's trunk may also be an important visual cue in male mate choice, reflecting the number of eggs available for transfer at any given time. While body weight does not appear to reflect the stage of development of female eggs in H. abdominalis (Poortenaar et al. 2004), weight may indicate the number of hydrated eggs a female has available for transfer and may possibly serve as an important mating cue to males. As larger stimulus individuals in trials were always both longer and heavier than smaller individuals, our data do not allow for an explicit investigation of this question here. While a general preference for larger-bodied individuals was shown by males, this preference was not influenced by either relative length or weight of the stimulus animals, and the length-based GLM model fit the data better than that based on weight.

Large females spent more time in the preference zones than small females. These behaviors may reflect increased reproductive interest or generally higher levels of activity in larger individuals. Most conspicuous was the complete lack of interest of the five smallest females in this study. While it is possible that these individuals had not yet reached maturity, sexual maturity in $H$. abdominalis typically occurs at a SL of $11 \mathrm{~cm}$ (Woods 2000), much smaller than that of the smallest female used here $(18.1 \mathrm{~cm})$. While female receptivity may be influenced by their own reproductive 
state (Jennions and Petrie 1997), egg development in female seahorses is continuous, and hydration of eggs can occur within only a few hours (Woods 2003), suggesting that variation in female receptivity should not have influenced our results.

This positive correlation between female body size and reproductive activity may have had an influence on the outcome of the focal-male trials, as courtship behavior in seahorses involves a close interaction between the sexes (see above). This makes it difficult to disentangle whether male preferences for large-bodied females reflect preferences for body size itself or for behavioral modifications associated with large size. Video-playback experiments offer a promising approach for isolating the motivations for reproductive behavior in seahorses (Kunzler and Bakker 2001). In video-playback experiments, live stimulus animals are replaced with video recordings, allowing the subtle manipulation of particular morphological traits without influencing behavior. Video-playback experiments have proven successful in the broad-nosed pipefish (S. typhle; Robinson-Wolrath 2006), and the use of this approach in seahorses may offer a means to determine the relative importance of female body size and reproductive behavior in male mating preferences.

Due in large part to the lower activity levels of females relative to males, the final sample size of females used in our tests of body size preferences was significantly reduced (14 females failed to show any activity compared to seven males). In an effort to only include individuals showing clear interest in reproduction, our sample was further reduced to include only those animals that visited both sizes of the experimental arena and displayed courtship behavior. From an initial 29 focal-female and 30 focal-male experimental trials, we ultimately included only ten female and 16 male trials in our final analyses. This sex difference in sample size may partially explain the difference in male and female behavior inferred here. Although based on an even smaller number of trials $(n=5)$, a recent study of Hippocampus guttulatus also found an absence of female preference for partners of large body size (Naud et al. 2009). While studies on both $H$. abdominalis and $H$. guttulatus have found evidence for differences in male and female reproductive activity, future studies of seahorse reproductive behavior should aim to include larger and more balanced sample sizes in order to minimize the possibility of statistical artifacts. Unfortunately, the current conservation status of seahorses imposes significant limitations on our ability to study the reproductive ecology of this group. We have used captive-reared animals in the present study, and a similar approach may allow the use of larger sample sizes in future research.

The results presented here highlight the feasibility of two-sided, two-choice experimental designs for the as- sessment of seahorse mating behavior. While we explicitly addressed the question of the role of body size in matechoice decisions, our experiment did not control for the influence of behavioral, olfactory, and/or other morphological characters on mate-choice decisions in H. abdominalis. Future experiments should attempt to isolate the role of olfactory and visual cues in reproduction decisions of seahorses through the use of pheromone-based trials and video-playback methods. It will be important to follow up the controlled preference trials outlined here with a second series of mating trials allowing direct interaction among animals in order to determine whether behavioral preferences accurately reflect reproductive decisions.

The results of the present study have identified striking differences in courtship behavior between male and female seahorses, with highly discriminatory males and indiscriminate females. Observations of male mate choice and femalefemale mating competition in wild populations of $H$. abdominalis (Wilson and Martin-Smith 2007) are consistent with sex-role reversal, and our results indicate that male preference for large partners may be influencing sexual size dimorphism observed in the wild. Interestingly, Woods (2000) found evidence of male-male competition in a large laboratory population of $H$. abdominalis. Taken together, these data suggest that the reproductive ecology of this species is plastic and may vary depending on local sex ratios and/or environmental conditions (Forsgren et al. 2004). While our experiment has demonstrated a clear difference in male and female preferences on the basis of body size, mate-choice decisions typically involve the integration of various sources of information, and future studies should investigate the relative importance of behavioral and chemical cues in mate-choice decisions of seahorses. The further refinement of the experimental design outlined here should offer a means to explore the factors responsible for sex-role flexibility in wild populations of seahorses, through the manipulation of sex-ratios and/or environmental parameters under controlled conditions.

Acknowledgments Thanks to Iris Eigenmann, Valeria Rispoli, and Kai Stölting for assistance with animal care and to Kerstin Musolf for comments on the manuscript. Funding for this work was provided by the University of Zurich. The experiments described here followed the ethical guidelines of the Veterinäramt Zürich (permit 185/2006).

\section{References}

Andersson M (1994) Sexual selection. Princeton University Press, Princeton

Berglund A, Rosenqvist G, Robinson-Wolrath S (2006) Food or sexmales and females in a sex role reversed pipefish have different interests. Behav Ecol Sociobiol 60:281-287 
Bergstrom CT, Real LA (2000) Towards a theory of mutual mate choice: lessons from two-sided matching. Evol Ecol Res 2:493-508

Blanckenhorn WU (2005) Behavioral causes and consequences of sexual size dimorphism. Ethology 111:977-1016

Burnham KP, Anderson DR (1998) Model selection and inference: a practical information-theoretic approach. Springer, New York

Byrne PG, Rice WR (2006) Evidence for adaptive male mate choice in the fruit fly Drosophila melanogaster. Proc R Soc Lond Ser B Biol Sci 273:917-922

Emlen ST, Oring LW (1977) Ecology, sexual selection, and the evolution of mating systems. Science 197:215-223

Forsgren E, Amundsen T, Borg AA, Bjelvenmark J (2004) Unusually dynamic sex roles in a fish. Nature 429:551-554

Foster SJ, Vincent ACJ (2004) Life history and ecology of seahorses: Implications for conservation and management. J Fish Biol 65:1-61

Herdman EJE, Kelly CD, Godin JGJ (2004) Male mate choice in the guppy (Poecilia reticulata): Do males prefer larger females as mates? Ethology 110:97-111

Jennions MD, Petrie M (1997) Variation in mate choice and mating preferences: a review of causes and consequences. Biol Rev Camb Philos Soc 72:283-327

Johnstone RA, Reynolds JD, Deutsch JC (1996) Mutual mate choice and sex differences in choosiness. Evolution 50:1382-1391

Jones AG, Moore GI, Kvarnemo C, Walker D, Avise JC (2003) Sympatric speciation as a consequence of male pregnancy in seahorses. Proc Natl Acad Sci U S A 100:6598-6603

Kokko H, Johnstone RA (2002) Why is mutual mate choice not the norm? Operational sex ratios, sex roles and the evolution of sexually dimorphic and monomorphic signalling. Philos Trans R Soc Lond B Biol Sci 357:319-330

Kolm N (2004) Female courtship in the Banggai cardinalfish: honest signals of egg maturity and reproductive output? Behav Ecol Sociobiol 56:59-64

Kunzler R, Bakker TCM (2001) Female preferences for single and combined traits in computer animated stickleback males. Behav Ecol 12:681-685

Kvarnemo C, Moore GI, Jones AG (2007) Sexually selected females in the monogamous Western Australian seahorse. Proc R Soc Lond Ser B Biol Sci 274:521-525

Lourie S (2003) Measuring seahorses. 4. University of British Columbia Fisheries Centre, Vancouver, p 15

Lourie SA, Vincent ACJ, Hall HJ (1999) Seahorses: an identification guide to the world's species and their conservation. Project Seahorse, London

Martin-Smith KM, Vincent ACJ (2005) Seahorse declines in the Derwent estuary, Tasmania in the absence of fishing pressure. Biol Conserv 123:533-545
Masonjones HD, Lewis SM (1996) Courtship behavior in the dwarf seahorse, Hippocampus zosterae. Copeia 1996:634-640

Naud MJ, Curtis JMR, Woodall LC, Gaspar MB (2009) Mate choice, operational sex ratio, and social promiscuity in a wild population of the long-snouted seahorse Hippocampus guttulatus. Behav Ecol 20:160-164

Poortenaar CW, Woods CMC, James PJ, Giambartolomei FM, Lokman PM (2004) Reproductive biology of female big-bellied seahorses. J Fish Biol 64:717-725

Robinson-Wolrath SI (2006) Video playback versus live stimuli for assessing mate choice in a pipefish. Env Biol Fish 75:409-414

Rohlf FJ (2004) tpsDIG, Version 2.05. SUNY, Stony Brook

Shuster SM, Wade MJ (2003) Mating systems and strategies. Princeton University Press, Princeton

Stölting KN, Wilson AB (2007) Male pregnancy in seahorses and pipefish: beyond the mammalian model. BioEssays 29:884-896

Trivers RL (1972) Parental investment and sexual selection. In: Campbell B (ed) Sexual selection and the descent of man, 1st edn. Heinemann, London, pp 136-179

Vincent ACJ (1990) Reproductive ecology of seahorses [Dissertation]. Cambridge University; $100 \mathrm{p}$

Vincent ACJ, Giles BG (2003) Correlates of reproductive success in a wild population of Hippocampus whitei. J Fish Biol 63:344-355

Vincent ACJ, Sadler RM (1995) Faithful pair bonds in wild seahorses, Hippocampus whitei. Anim Behav 50:1557-1569

Wilson AB, Martin-Smith KM (2007) Genetic monogamy despite social promiscuity in the pot-bellied seahorse (Hippocampus abdominalis). Mol Ecol 16:2345-2352

Wilson AB, Ahnesjö I, Vincent ACJ, Meyer A (2003) The dynamics of male brooding, mating patterns, and sex roles in pipefishes and seahorses (family Syngnathidae). Evolution 57:1374-1386

Woods CMC (2000) Preliminary observations on breeding and rearing the seahorse Hippocampus abdominalis (Teleostei: Syngnathidae) in captivity. NZ J Mar Freshwater Res 34:475-485

Woods CMC (2003) Effect of stocking density and gender segregation in the seahorse Hippocampus abdominalis. Aquaculture 218:167-176

Woods CMC (2005) Reproductive output of male seahorses, Hippocampus abdominalis, from Wellington Harbour, New Zealand: implications for conservation. NZ J Mar Freshwater Res 39:881888

Woods CMC (2007) Aquaculture of the big-bellied seahorse Hippocampus abdominalis Lesson 1827 (Teleostei: Syngnathidae) [Dissertation].Victoria University of Wellington; $\mathrm{p} 238$

Woods CMC, Martin-Smith KM (2004) Visible implant fluorescent elastomer tagging of the big-bellied seahorse, Hippocampus abdominalis. Fish Res 66:363-371 